\title{
NONUNIFORMIZABLE SKEW CYLINDERS. A COUNTEREXAMPLE TO THE SIMULTANEOUS UNIFORMIZATION PROBLEM
}

\begin{abstract}
A.A.Glutsuk
Abstract. At the end of 1960-ths Yu.S.Ilyashenko stated the problem: is it true that for any one-dimensional holomorphic foliation with singularities on a Stein manifold leaves intersecting a transversal disc can be uniformized so that the uniformization function would depend holomorphically on the transversal parameter? In the present paper we construct a two-dimensional counterexample. This implies existence of a counterexample given by a foliation on affine algebraic surface by level curves of a polynomial with singularities deleted. This implies also that Bers' simultaneous uniformization theorem [8] for regular holomorphic foliations by compact Riemann surfaces does not extend to the general case of foliations by compact Riemann surfaces with singularities.
\end{abstract}

\section{Introduction, main results and the history.}

It is well known that in the generic case the arrangement of phase curves of a polynomial vector field in $\mathbb{C}^{n}$ is quite complicated. For example, each phase curve of a generic polynomial vector field in $\mathbb{C}^{2}$ is everywhere dense in the phase space $[1,2,3]$.

The approach to the investigation of this complicated picture proposed by Yu.S.Ilyashenko was the uniformization of leaves (i.e., of phase curves). The result of the uniformization of a single leaf is well known. It is given by the classical theorem on the classification of simply connected Riemann surfaces. But it is important for the investigation of the foliation as a whole to know the dependence of the uniformizing function on the parameter.

In the late 1960-ths Ilyashenko stated the problem: is it true that the leaves of a one-dimensional holomorphic foliation with singularities on a smooth affine algebraic manifold (or more generally, Stein manifold) admit a uniformization such that the uniformizing function depends on the parameter holomorphically? Later in 1972 he proved the theorem asserting that the union of universal coverings over the leaves with marked initial points on a transversal disk is a Stein manifold (see [4]).

In the present paper we prove a negative answer to the general Ilyashenko problem (Theorem 1 and Corollaries 1,2).

Research supported by CRDF grant RM1-229, by INTAS grant 93-0570-ext, by Russian Foundation for Basic Research (RFBR) grant 98-01-00455, by State Scientific Fellowship of Russian Academy of Sciences for young scientists, by the European Post-Doctoral Institut joint fellowship of Max-Planck Institut für Mathematik (Bonn) and IHÉS (Bures-sur-Yvette, France) 
Remark 1. Ilyashenko problem remains open, e.g., for holomorphic vector fields in $\mathbb{C}^{2}$ and foliations by analytic curves in $\mathbb{C P}^{2}$ (cf. Question 1).

To state the main results, let us recall some Definitions.

Definition 1. Let $S$ be a complex manifold, $\Sigma \subset S$ be a closed subset of complex codimension at least 2. Let $F$ be a one-dimensional holomorphic foliation on $S \backslash \Sigma$ (i.e., a holomorphic tangent line field) that extends analytically to no point of $\Sigma$. Then one says that $F$ is a one-dimensional holomorphic foliation on $S$ with the singular set $\Sigma$ (or a one-dimensional holomorphic foliation with singularities on $S$ ).

Example 1. Let $S$ be a quasiprojective surface. Each nonconstant rational function on $S$ defines a one-dimensional holomorphic foliation on $S$ by its level curves with singularities deleted.

Definition 2. Let $S$ be a complex manifold, $F$ be a one-dimensional holomorphic foliation with singularities on $S$. Let $D$ be an embedded disc in $S$ transversal to $F$. The universal covering manifold associated to the triple $(S, F, D)$ is the union of the universal coverings over the leaves intersecting $D$ with marked points in $D$. More precisely, it is the set of all the triples consisting of a point $z \in D$, a point $z^{\prime}$ of the leaf containing $z$, and a homotopy class of a path connecting $z$ to $z^{\prime}$ in the leaf.

Remark 2. In the condition of the previous Definition let $S$ be Stein. Then for any transversal disc $D$ the correspondent universal covering manifold admits a natural structure of complex manifold and is Stein. This was proved by Ilyashenko [4].

Definition 3. Let $D$ be unit disc in complex line (or more generally, a polydisc), $M$ be a complex manifold, $p: M \rightarrow D$ be a proper holomorphic surjection having nonzero derivative. Say that the triple $(M, p, D)$ is a skew cylinder with the base $D$, if

1) all the level sets of the map $p$ are simply connected holomorphic curves;

2) the "fibration" $M$ possesses a section, that is, there exists a holomorphic mapping $i: D \rightarrow M$ such that $p \circ i=I d$.

Example 2. In the condition of Definition 2 let $S$ be either Stein, or a projective manifold. Then the correspondent universal covering manifold is a skew cylinder. This follows from existence of a natural structure of complex manifold on the universal covering manifold. In the Stein case this statement was proved in [4] (cf. Remark 2). In the projective case it was proved by myself and a bit later by Chirka (in unpublished papers).

Definition 4. A skew cylinder $(M, p, D)$ is said to be uniformizable, if there exists a biholomorphic mapping $u: M \rightarrow \overline{\mathbb{C}} \times D$ (not necessarily "onto") that forms a commutative diagram with the projections to $D$.

Theorem 1. There exists a two-dimensional nonuniformizable Stein skew cylinder.

Theorem 1 is proved in Subsection 2.

Corollary 1. 1 There exist $n \in \mathbb{N}$, a smooth affine algebraic surface $S \subset \mathbb{C}^{n}$ $(\operatorname{dim} S=2)$ and a polynomial $P$ in $\mathbb{C}^{n},\left.P\right|_{S} \not \equiv$ const such that there exists a nonuniformizable skew cylinder $(M, p, D)$ in $S(M \subset S)$ with the projection $P$.

\footnotetext{
${ }^{1}$ The argument yielding reduction of Corollary 1 from Theorem 1 was given in an unpublished paper by Ilyashenko (late $1960^{t h}$ ). It was independently obtained by the author (see Subsection $3)$.
} 
The proof of Corollary 1 is presented in Subsection 3.

Corollary 2. In the conditions of Corollary 1 let $i(D)$ be the image of the section of the cylinder $M$. Let $\widetilde{M}$ be the universal covering manifold associated to $S$ (or its Zariski closure $\bar{S} \subset \mathbb{C P}^{n}$ ), to the foliation $P=$ const (its extension to $\bar{S}$ ), and to the embedded disc $i(D)$. Then $\widetilde{M}$ is nonuniformizable.

Remark 3. In 1960 L.Bers [8] proved that the tautological fibration by Riemann surfaces over Teichmüller space is simultaneously uniformizable. Corollary 2 implies that in general, this is not the case for the tautological fibration by curves with singularities over disc embedded in the Deligne-Mumford compactification of the moduli space.

Question 1. Does there exist a polynomial (holomorphic) vector field in $\mathbb{C}^{2}$ (or a foliation by analytic curves in $\mathbb{C P}^{2}$ with isolated singularities) and a transversal disc such that the correspondent universal covering manifold is not uniformizable?

The simultaneous uniformization problem was studied by Yu.S.Ilyashenko and A.A.Shcherbakov [5]. They proved that if a skew cylinder with a smooth striclty pseudoconvex boundary is uniformizable, then this remains valid for skew cylinders obtained by small perturbation of its complex structure. Shcherbakov proved (in the same paper) that each Stein skew cylinder can be exhausted by skew subcylinders with strictly pseudoconvex boundaries.

\section{Proof of Theorem 1.}

The proof of Theorem 1 is based on the following joint result by Berndtsson and Ransfold [6].

Theorem 2 [6]. Let $D$ be unit disc with the coordinate $z, p$ be the standard projection to $D$ of the direct product $\mathbb{C} \times D$. Let $E_{+}=\left\{\frac{1}{2}, \frac{n}{2 n+1}\right\}_{n \in \mathbb{N}}, E_{-}=-E_{+} \subset D$. There exists a closed subset $K \subset \mathbb{C} \times D$ such that

1) the complement $M^{\prime}=\mathbb{C} \times D \backslash K$ is pseudoconvex;

2) for any $z \notin E_{+} \cup E_{-}$the fiber $K \cap p^{-1}(z)$ is a disc;

3) for any $z \in E_{+} K \cap p^{-1}(z)=0 \times z$;

4) for any $z \in E_{-} K \cap p^{-1}(z)=1 \times z$.

For the completeness of presentation, we recall the construction of the set $K$ from [6]. Let $w$ be the coordinate in the fiber $\mathbb{C}$ in the direct product $\mathbb{C} \times D$. Let $u(z)=\sum_{n=1}^{+\infty} 2^{-n}\left(\ln \left|z-\frac{n}{2 n+1}\right|+\ln \left|z+\frac{n}{2 n+1}\right|\right), A \in \mathbb{R}^{+}$. The function $u$ is harmonic and is equal to $-\infty$ at $E_{ \pm}$. Let $f: D \rightarrow \mathbb{C}$ be a $C^{\infty}$ function with bounded derivatives (up to the second order) that is holomorphic in a neighborhood of the union $E_{+} \cup E_{-}$so that $\left.f\right|_{E_{+}}=0,\left.f\right|_{E_{-}}=1$. Define

$$
K=\left\{|w-f(z)| \leq e^{u(z)+|z|^{2}+A}\right\}
$$

The fibers of $K$ over $E_{+}\left(E_{-}\right)$are single points where the coordinate $w$ is equal to 0 and 1 respectively. If $A$ is large enough, then $\mathbb{C} \times D \backslash K$ is pseudoconvex. The proof of this statement (presented in [6]) is a straightforward calculation of the Levi form together with the argument on approximation of the harmonic function $u$ by decreasing sequence of smooth subharmonic functions.

The nonuniformizable skew cylinder we are looking for is the universal covering over the complement $M^{\prime}=\mathbb{C} \times D \backslash K$ (denote this covering by $M$ ). Equivalently, 
it is the universal covering manifold for the foliation on $M^{\prime}$ by the level curves of the projection to $D$ (all these level curves intersect one and the same transversal $\operatorname{dics} N \times D$ correspondent to $N$ large enough).

Indeed, $M$ is a Stein skew cylinder. This follows from its previous characterization as the universal covering manifold, the fact that $M^{\prime}$ is Stein (it is pseudoconvex by Theorem 2) and Ilyashenko's theorem [4] (cf. Remark 2). Let us prove that $M$ is nonuniformizable by contradiction. Suppose the contrary, i.e., there exists a uniformization $u: M \rightarrow \overline{\mathbb{C}} \times D$. Let $\pi: \overline{\mathbb{C}} \times D \rightarrow \overline{\mathbb{C}}$ be the standard projection, $f=\pi \circ u$ be the correspondent coordinate component of $u$. The fibers of the cylinder $M$ over $E_{ \pm}$are conformally equivalent to complex plane. Let $w$ be the coordinate in $\mathbb{C}$ (we consider it as a coordinate on $M^{\prime}$ ). Consider the chart $\ln w$ on the fibers of $M$. It is a well-defined 1-to- 1 chart on the fibers over $E_{+}$, and this is not the case for the fibers over $E_{-}$, where this chart is multivalued and has branch points. Therefore, for any $z \in E_{+}$the restriction to the fiber over $z$ of the function $f$ (which is univalent) is Möbius in the chart $\ln w$, and this is not the case for the fibers over $E_{-}$.

Let $S f$ be the Schwartzian derivative of the function $f$ in the coordinate $\ln w$. It is a holomorphic function on $M$. It vanishes at the fibers of $M$ over $E_{+}$and does not vanish identically on the union of the fibers over $E_{-}$by the previous statement. The first one of the two last statements implies that $S f \equiv 0$ (the set $E_{+}$contains the limit point $\frac{1}{2}$ ), which contradicts the second statement. This proves Theorem 1 .

3. Proof of Corollary 1. Let us sketch the reduction of Corollary 1 from Theorem 1. To do this, let us recall the following

Definition 5. Let $(M, p, D)$ be a skew cylinder, $B \subset M$ be its subdomain. Then $B$ is called subcylinder, if the triple $(B, p, p(B))$ is a skew cylinder.

The reduction of Corollary 1 from Theorem 1 is based on the two following statements.

Theorem 3 (A.A.Shcherbakov, [5]). Any Stein skew cylinder is exhausted by increasing sequence of subcylinders with compact closures and strictly pseudoconvex boundaries.

Proposition 1. Let a Stein skew cylinder be exhausted by increasing sequence of uniformizable subcylinders. Then it is uniformizable.

The proof of Proposition 1 is similar to the proof of the analogous statement from [5] and is omitted to save the space.

Corollary 3. Any nonuniformizable Stein skew cylinder contains a nonuniformizable subcylinder with compact closure.

Corollary 1 is implied by Corollary 3 and the following

Lemma 1. Let $(M, p, D)$ be a Stein skew cylinder, $M$ be embedded as a submanifold in $\mathbb{C}^{n}, B$ be its subcylinder with compact closure. There exist an affine algebraic surface $S \subset \mathbb{C}^{n}$ and a polynomial $P$ in $\mathbb{C}^{n},\left.P\right|_{S} \not \equiv$ const, and a biholomorphic mapping $B \rightarrow S$ that forms a commutative diagram with $p$ and $P$.

Let us reduce Corollary 1 from Lemma 1 . Let $(M, p, D)$ be a nonuniformizable Stein cylinder, $M \subset \mathbb{C}^{n}$. Let $B \subset M$ be a nonuniformizable subcylinder with 
compact closure. Then the correspondent surface $S$ and polynomial $P$ from Lemma 1 satisfy the statements of Corollary 1 . This proves Corollary 1 modulo Lemma 1.

Proof of Lemma 1.

Let $(M, p, D), M \subset \mathbb{C}^{n}, B$ be as in Lemma 1. Let us construct a smooth affine algebraic surface $S \subset \mathbb{C}^{n}$ satisfying the statements of Lemma 1 .

The surface $M$ is a complete intersection: there exist $n-2$ holomorphic functions $f_{1}, \ldots, f_{n-2}$ in $\mathbb{C}^{n}$ such that $M$ is the transversal intersection of their zero level hypersurfaces. This follows from simple connectivity of $M$ (by definition) and results of the paper [7]. Let $i: D \rightarrow M$ be the section of $M$. The projection $p: M \rightarrow D$ extends up to a holomorphic function on $\mathbb{C}^{n}$ by classical theorem on extension of analytic function from Stein submanifold. Analogously, the section image $i(D)$ is zero set of a holomorphic function on $M$ with multiplicity 1 . This function has an extension to $\mathbb{C}^{n}$ (let us fix this extension and denote it by $g$ ).

Let us fix another subcylinder $B^{\prime} \subset M$ containing $B$ with compact closure and strictly pseudoconvex boundary (its existence follows from Theorem 3). In the proof of Lemma 1 we use the fact that the functions $f_{i}, i=1, \ldots, n-2$, and $p$ can be approximated by polynomials uniformly on each ball (in particular, in a one containing $B^{\prime}$, denote the correspondent polynomials by $F_{i}$ and $P$ ). Let us fix these polynomials and denote by $S$ the irreducible component of the conjoint zero set of the polynomials $F_{i}$. Let us prove the statement of Lemma 1 for a subcylinder $B^{\prime \prime} \subset M$ close to $B^{\prime}$, provided that the previous approximations are accurate enough. To do this, we use the following

Theorem 4. Let $(M, p, D)$ be a Stein cylinder, $B \subset M$ be its subcylinder with compact closure and strictly pseudoconvex boundary. There exist $R, r \in \mathbb{N}$ (independent on the cylinders) with the following property: for any $\varepsilon>0$ and any $C^{R}$ - small (dependently on $\varepsilon$ ) perturbation of the standard complex structure on $\bar{B}$ such that $p$ remains holomorphic there exists a mapping $f: B \rightarrow M$ holomorphic with respect to the perturbed complex structure on $B$ commuting with $p$ such that $\operatorname{dist}_{C^{r}}(f, \mathrm{Id})<\varepsilon$.

Theorem 4 was proved in [5] in the particular case, when $M=\mathbb{C} \times D$. The proof remains valid with obvious changes in the general case.

Let us construct the cylinder $B^{\prime \prime} \subset S$ approximating $B^{\prime}$ (see the paragraph preceding Theorem 4). To do this, we show that there exists a $C^{\infty}$ mapping $f: \overline{B^{\prime}} \rightarrow S$ close to identity (dependently on the accuracy of the previous approximations of functions by polynomials) that transforms the section of the cylinder $B^{\prime}$ to a holomorphic curve and forms a commutative diagram with the projections $\left.p\right|_{M}$ and $\left.P\right|_{S}$. Then the image $f\left(B^{\prime}\right)$ is a skew cylinder with the projection $P$, and the pullback of its standard complex structure under $f$ is a perturbation of the complex structure of $B^{\prime}$. By Theorem $4, B^{\prime \prime}$ is biholomorphically equivalent to a subcylinder in $M$ close to $B^{\prime}$, provided that the previous approximations are accurate enough. This will prove Lemma 1.

To construct the mapping $f$, consider the holomorphic "section" $\phi: D \rightarrow S$ inverse to the projection $P$ and close to the section $i$, e.g., defined by the condition that for any $z \in D \phi(z) \in\{g=0\}$ (recall, that the hypersurface $g=0$ is transversal to $M$ and contains the section image $i(D))$. For any $z \in D$ by $L_{z}$ denote the fiber over $z$ of the cylinder $B^{\prime}$. The mapping $f$ is defined as follows: $f(i(z))=\phi(z) ; f$ maps the fiber $L_{z}$ into the lever curve $\{P=z\} \cap S$ and $f^{-1}$ is the normal projection 
to $L_{z}$ of the tubular neighborhood of $L_{z}$ in $\mathbb{C}^{n}$. The map $f$ is well-defined and close to identity, if the initial approximations of the functions by polynomials are accurate enough. Lemma 1 is proved.

\section{ACKNOWLEDGEMENTS}

I am grateful to Yu.S.Ilyashenko who attracted my attention to the problem. I am grateful to G.M.Henkin, who informed me about the paper [6] (one of results from [6] became the base for the proof). I wish to thank both them and I.Lieb, B.Hill, R.Gunning, M.Gromov and S.Nemirovskii for helpful discussions.

\section{REFERENCES}

1. Arnold, V.I. and Ilyashenko Yu.S., Ordinary Differential Equations. - Itogi Nauki i Techniki, Contemporary Problems in Mathematics, Fundamental Directions, Moscow: VINITI, 1985, vol.1, part 1.

2. Muller, B., On the Density of the solutions of Certain Differential Equations in $\mathbb{C P}^{n}$. - Mat. Sb., 1975 , vol.98, no. 3, pp. 363-377.

3. Khudai-Verenov, M.O., On a property of the Solutions of a Differential Equation. - Mat. Sb., 1962, vol.56 (98), no.3, p.301-308.

4. Ilyashenko, Yu.S., Foliations by analytic curves. - Mat. Sb., 88, No. 4, $558-577$ (1972).

5. Ilyashenko, Yu.S. and Shcherbakov, A.A., Skew Cylinders and Simultaneous Uniformization. - Proc. of Steklov Math. Inst., 1996, vol.213.

6. Berndtsson, B.; Ransfold, T.J. Analytic multifunctions, the $\bar{\partial}$ - equation, and a proof of the corona theorem.- Pacific J.Math. 124 (1986) No 1, 57-72.

7. Forster, O.; Bănică, C. Complete intersections in Stein Manifolds. - Manuscripta Math. 37 (1982), no. 3, 343-356.

8. Bers, L.; Simultaneous uniformisation. - Bull. Amer. Math. Society. 66 (1960) 94-97. 